\title{
Hepatic Alveolar Hydatid Cyst: A Brief Review of Published Cases from Iran in the Last 20 Years
}

\author{
Bita Geramizadeh, ${ }^{1,2, *}$ and Mohammad Baghernezhad ${ }^{1}$ \\ ${ }^{1}$ Department of Pathology, Shiraz University of Medical Sciences, Shiraz, IR Iran \\ ${ }^{2}$ Transplant Research Center, Shiraz University of Medical Sciences, Shiraz, IR Iran \\ "Corresponding author: Bita Geramizadeh, Department of Pathology, Shiraz University of Medical Sciences, Shiraz, IR Iran. Tel/Fax: +98-7136473238, E-mail: \\ geramib@gmail.com,geramib@sums.ac.ir
}

Received 2016 May 03; Revised 2016 June 13; Accepted 2016 September 04.

\begin{abstract}
Context: Echinococcus multilocularis is the cause of alveolar hydatid disease, which most commonly involves the liver in more than $90 \%$ of cases. This disease is endemic in northern Iran. However, there are very few published cases from Iran. In this article, we will review all of the published cases of hepatic alveolar echinococcosis from Iran regarding all aspects, including clinical, paraclinical, and treatment protocols.

Evidence Acquisition: In this brief review, the published cases of hepatic Alveolar Echinococcosis (AE) from Iran were retrieved for review via a search in PubMed, Scopus, Google Scholar, IranMedex, scientific information database (SID), Magiran, and Irandoc (1995 - 2015) using the keywords Echinococcus multilocularis and Iran, Alveolar hydatid cyst and Iran, liver and Alveolar hydatid cyst and Iran, and Hepatic alveolar hydatid cyst and Iran. The following inclusion criteria were employed: 1, articles must be written in English or Farsi; 2, articles must have been published between 1995 and 2015; 3) cases must have been confirmed by pathological diagnosis.

Results: There were 24 published cases of liver-involved alveolar hydatid cyst from Iran. The disease was more common in young-to middle-aged women in northeast Iran. The most common presenting signs and symptoms were abdominal pain with hepatomegaly and liver mass. Most of the patients were treated by surgery and albendazole. The few unresectable liver masses were treated by medical therapy. No liver transplantation for this disease was reported from Iran.

Conclusions: Hepatic alveolar hydatid cyst should be considered one of the important differential diagnoses of liver masses, especially in endemic areas of the world.
\end{abstract}

Keywords: Alveolar Hydatid Cyst, Liver, Alveolar Echinococcosis, Echinococcosis multilocularis

\section{Context}

Echinococcus multilocularis is one of the rare types of parasitic infestations in the northern hemisphere which can involve several organs, especially the liver. Iran has been known as one of the endemic areas in the world. However, there is not enough information about liver involvement related to this parasite in human beings (Hepatic Alveolar Echinococcosis) (1). Liver involvement of this parasite is a life-threatening infestation, especially when diagnosis is delayed, which itself can be life-threatening (2). In this review, we discuss all of the published cases of hepatic AE from Iran in regard to epidemiology, clinical presentation, diagnosis, and protocols of treatment.

\subsection{Echinococcus Multilocularis: Classification and Life Cycle}

E. multilocularis is classified as a small cestode. The adult form of this tapeworm lives in the intestines of natural definitive hosts, which can be wild carnivores such as red fox or domestic dogs and cats. The eggs released by the adult tapeworm are passed in the stool of the definitive hosts, which then is ingested by the intermediate hosts, such as small rodents or red foxes. However, there are other accidental intermediate hosts, such as humans. Ingestion of the eggs by humans usually occurs because of the contamination of food or water by the fecal material of definitive hosts. After the ingestion of the eggs by humans, the larvae (metacestodes) travel to the liver, where they create alveolar small vesicles and alveolar cysts (3-5).

\subsection{Hepatic Alveolar Cyst (Hepatic AE)}

As mentioned, hepatic AE is caused by the infestation of humans, as an accidental intermediate host, by the larval stage of E. multilocularis. The echinococcal metacestodes grow in the liver and create an alveolar-like structure, which consists of several vesicles surrounded by large 
granuloma. Human $\mathrm{AE}$ is a severe and emerging disease which, with delayed diagnosis, can be life-threatening (2).

\subsection{Epidemiology of Hepatic $A E$}

$\mathrm{AE}$ is considered one of the most dangerous parasitic infestations of human beings in the northern hemisphere. New AE cases in Western and central Europe have been estimated in the range of 170 - 200 per year. The highest reported numbers of AE from Europe were from France, Germany, Switzerland, Lithuania, and Poland, and it is extremely rare in the eastern and central parts of Europe (6).

High parasite prevalence is also reported in the Middle East and Arabic North Africa. In the Middle East, Iran, Iraq, and Turkey are known as areas where AE is endemic (7). However, from these regions, where the parasite is known to be present, there is a scarcity of information about recent distribution and frequency (8).

\section{Evidence Acquisition}

In this brief review, the published cases of hepatic $\mathrm{AE}$ from Iran were retrieved for review via a search in PubMed, Scopus, Google Scholar, IranMedex, scientific information database (SID), Magiran, and Irandoc (1995 - 2015), using the keywords Echinococcus multilocularis and Iran, Alveolar hydatid cyst and Iran, liver and Alveolar hydatid cyst and Iran, and Hepatic alveolar hydatid cyst and Iran.

The following inclusion criteria were employed: 1, articles must be written in English or Farsi; 2, articles must have been published between 1995 and 2015; 3, cases must have been confirmed by pathologic examination.

\section{Results}

During the 20 years between 1995 and 2015, there were 24 published cases of hepatic AE from Iran (9-14). There were 20 female patients and 4 male $(F / M=5 / 1)$. The age range of these patients was 21 - 74 years $(42.6 \pm 12.2)$. Twenty-two patients were from the northeast part of the country, i.e., Khorasan Razavi province, one patient was from Ardebil province, and one case was reported from the south, i.e., Khuzestan province.

Clinical presentation of the patients was mostly right upper quadrant (RUQ) pain in 20 (83\%) patients, anorexia and weight loss in 2 (8.3\%) patients, jaundice in 5 (20.8\%) patients, and fever in $4(16.6 \%)$ patients. One case was incidentally detected during surgery for a uterine fibroma (13).

The most common sign in physical examination was hepatomegaly and liver mass in all 24 patients, which was tender in two of them (8.3\%). One patient presented with cough, bloody sputum, and fatigue, secondary to lung metastasis (13). Duration of the disease at the time of diagnosis was from 20 days to 5 years ( $35.6 \pm 27.5$ months).

The most common imaging modality used in the reported cases was abdominal ultrasonography. In all of the cases, sonographic findings were described as large (from $10-30 \mathrm{~cm}$ ) solid or cystic liver masses with heterogeneous echogenicity and irregular margins. Magnetic resonance image (MRI) and CT scan, used in a few cases, revealed large multiloculated liver masses with heterogeneous density and irregular and infiltrative margin. Calcification was reported in one case. In the same case, para-aortic lymphadenopathy was detected (11).

Fifteen cases had a mass in the right lobe of the liver, seven cases in the left lobe, and in two patients, there were multiple liver masses in both liver lobes. In most of the published cases, white blood cell count (WBC) was not reported. However, in four reported cases, WBC was increased in two of the patients $(5,200-15,500 / \mathrm{mL})$. Liver enzymes, including alanine aminotransferase (ALT), aspartate aminotransferase (AST), and alkaline phosphatase (ALP) were mostly abnormal. ALT was 10 - 268 IU/L (74.9 土 78.6) (normal < $28 \mathrm{IU} / \mathrm{L})$, AST was 18 - $262 \mathrm{IU} / \mathrm{L}(79.83 \pm 80.4)$ (normal < $28 \mathrm{IU} / \mathrm{L}$ ), and ALP was $90-1919 \mathrm{IU} / \mathrm{L}(578.7 \pm 595)$ (normal $<150 \mathrm{IU} / \mathrm{L}$ ).

Bilirubin level was reported in 21 patients as between 0.3 to $29.3 \mathrm{mg} / \mathrm{dL}(6.5 \pm 8.7)$ with a normal of $<1.2 \mathrm{mg} / \mathrm{dL}$. Most of the patients had a normal bilirubin level. Table 1 shows the primary characteristics of the 24 patients with hepatic AE published from Iran.

Table 1. Primary Characteristics of 24 Published Cases of Hepatic Alveolar Hydatid Cyst From Iran

\begin{tabular}{|c|c|}
\hline $\mathbf{F} / \mathbf{M}$ & $20 / 4$ \\
\hline Age & $21-74(42.6 \pm 12.20)$ \\
\hline Duration of symptoms & 20 days to 5 years ( $35.6 \pm 27.5$ months) \\
\hline Clinical presentation & Abdominal (RUQ) pain \\
\hline Physical examination & Hepatomegaly and liver mass \\
\hline Location & Khorasan Province \\
\hline Lobe of involvement & Right lobe \\
\hline Size & $10-30 \mathrm{~cm}$ \\
\hline Bilirubin, mg/dL & 0.3 to $29.3 \mathrm{mg} / \mathrm{dL}(6.5 \pm 8.7)$ \\
\hline ALT, IU/L* & $10-268 \mathrm{IU} / \mathrm{L}(74.9 \pm 78.6)$, normal $<28 \mathrm{IU} / \mathrm{L}$ \\
\hline AST, IU/L** & $18-262 \mathrm{IU} / \mathrm{L}(79.83 \pm 80.4)$ \\
\hline ALP, IU/L $\mathbf{L}^{* * *}$ & $90-1919(578.7 \pm 595)$ \\
\hline
\end{tabular}

The gold standard for final diagnosis in all of the cases 
of hepatic AE from Iran was tissue biopsy and pathologic diagnosis, either with a wedge biopsy or needle biopsy. Most of the cases were treated by a combination of surgery and antiparasitic medical therapy, i.e., albendazole, for six months to two years.

Nine cases had large, unresectable liver masses, one of which was scheduled for liver transplantation, but the patient did not accept it. However, she responded to medical therapy and, after six months' follow-up, she remained alive $(9,10)$. Postoperative complications for patients who underwent resection included postsurgical abscess, cholangitis, and biliopulmonary fistula $(13,14)$. Death was reported in three patients, secondary to lung metastasis, infection, and fistula (13).

\section{Discussion}

Alveolar echinococcosis in humans results from infestation by the larval form of Echinococcus multilocularis. The larval form rapidly proliferates and produces an alveolarlike pattern of microvesicles in different tissues of the body, especially the liver (15). Therefore, the characteristic feature of $\mathrm{AE}$ is its tumor-like pattern of growth. The liver is the first and most common organ to be affected by larval infestation. Hepatic AE is a rare and life-threatening parasitic disease, which develops as a result of intrahepatic growth of the Echinococcus multilocularis (4). Liver involvement related to Echinococcus multilocularis was first observed by Virchow in 1855, who published an article entitled Echinococcus-tumor of the liver (16).

The WHO-IWGE PNM classification system was established in 2001, based on imaging findings, as an international standardization for evaluation of diagnostic and therapeutic modalities. $\mathrm{P}$ is the degree of extension of the parasite, $\mathrm{N}$ indicates the involvement of nearby organs, and $\mathrm{M}$ is considered metastasis. This classification was developed to evaluate the extension of the disease and therapeutic approaches (15).

$\mathrm{AE}$ is characterized by primary lesions in the liver, producing tumor-like, multi-vesicular, infiltrating structures. Early diagnosis of AE by characteristic signs and symptoms, imaging modalities, immunologic tests (such as ELISA), histopathological examination, or molecular analysis of tissue materials can be life-saving and will significantly improve the prognosis of AE patients. Recently developed and improved serological and molecular tests for AE have been shown to be reliable (17). However, in most patients, characteristic symptoms are not present, and in most geographic areas of world, as in many parts of Iran, these tests are not available.

Ultrasonography, CT scan, and MRI are the most common imaging modalities for the diagnosis of $\mathrm{AE}$ in the liver. Ultrasonography is the preferred method for the screening of AE in the liver. Large liver masses with mixed echogenicity and irregular margin are the most common sonographic findings (18). The most common imaging modality in the published cases from Iran was ultrasonography.

It is recommended to perform CT scans in cases where liver-involved AE is suspected, but in most of the published cases from Iran, no CT report was mentioned. Sensitivity of this method is more than $90 \%$. AE in CT presents with a hypodense liver mass, very similar to a malignant infiltrative process with irregular borders. CT scan has the capacity to determine the extension of the disease, as well as the number, size, and localization (12). Magnetic resonance imaging (MRI) is also a good modality to diagnose the extension of the disease. In MRI, the liver mass of AE presents as an irregular, solid-cystic mass, with honeycomb appearance in T2-weighted images (19).

Fluorodeoxyglucose positron emission tomography (FDG-PET) is a noninvasive modality for the detection of metabolic activity in AE. Delayed images would increase its sensitivity for the assessment of the metabolism of the lesion and its reliability for the continuation or withdrawal of antiparasitic treatment (20). PET-CT can be used to monitor suppression of periparasitic inflammatory activity and has an important role for excluding the metastatic spread. However, expensive and sophisticated equipment is not widely available, which limits its use for a routine procedure (19).

The gold standard for the diagnosis of hepatic AE is histopathology. In tissue, the most common presentation is extensive necrosis, with small vesicles and delicate membranes scattered in the necrosis. In our experience, the most common pitfall for the pathologist is missing the above-mentioned structures and calling the biopsy unsatisfactory, because of extensive necrosis $(9,10)$. This places emphasis on the role of the pathologist in the diagnosis of $\mathrm{AE}$ in the liver, because delayed diagnosis of hepatic $\mathrm{AE}$ leads to advanced disease with unresectable masses and poor prognosis (21). In these circumstances, for very large, unresectable masses, the only surgical therapeutic modality can be liver transplantation (22).

In addition to surgical approaches, considering longterm (six months to two years) medical antiparasitic therapy (e.g., albendazole) is very important to decrease the risk of recurrence and postsurgical complications (23).

According to the previous studies in the English literature, although liver hydatid cyst, secondary to Echinococcus granulosus, is a very common disease in Iran, very few cases of hepatic alveolar hydatid cyst have been published from Iran (12). Despite the rarity of this disease, delayed diagnosis can be very detrimental. Thus, it is very important 
to consider this disease in the differential diagnosis of patients with liver mass, especially those from endemic areas (e.g., northeast Iran) $(9,10)$.

\section{References}

1. Borji H, Emami MR, Maleki M, Razmi GH, Kazemi Mehrjerdi $H$ Moghaddas E. Alveolar echinococcosis infection in a monkey (Ateles geoffroyi) in Mashhad, Iran. Iran public health. 2012;41(2):111-6.

2. Nunnari G, Pinzone MR, Gruttadauria S, Celesia BM, Madeddu G, Malaguarnera G, et al. Hepatic echinococcosis: clinical and therapeutic aspects. World J Gastroenterol. 2012;18(13):1448-58. doi: 10.3748/wjg.v18.i13.1448. [PubMed: 22509076].

3. Moro P, Schantz PM. Echinococcosis: a review. Int J Infect Dis. 2009;13(2):125-33. doi: 10.1016/j.ijid.2008.03.037. [PubMed: 18938096].

4. Kantarci M, Bayraktutan U, Karabulut N, Aydinli B, Ogul H, Yuce I, et al. Alveolar echinococcosis: spectrum of findings at cross-sectional imaging. Radiographics. 2012;32(7):2053-70. doi: 10.1148/rg.327125708. [PubMed: 23150858].

5. Thompson RC. The taxonomy, phylogeny and transmission of Echinococcus. Exp Parasitol. 2008;119(4):439-46. doi: 10.1016/j.exppara.2008.04.016. [PubMed: 18539274].

6. Conraths FJ, Deplazes P. Echinococcus multilocularis: Epidemiology, surveillance and state-of-the-art diagnostics from a veterinary public health perspective. Vet Parasitol. 2015;213(3-4):149-61. doi: 10.1016/j.vetpar.2015.07.027. [PubMed: 26298509].

7. Sadjjadi SM. Present situation of echinococcosis in the Middle East and Arabic North Africa. Parasitol Int. 2006;55 Suppl:S197-202. doi: 10.1016/j.parint.2005.11.030. [PubMed: 16337429].

8. Jenkins DJ, Romig T, Thompson RC. Emergence/re-emergence of Echinococcus spp.-a global update. Int J Parasitol. 2005;35(11-12):120519. doi: 10.1016/j.ijpara.2005.07.014. [PubMed:16157340]

9. Geramizadeh B, Nikeghbalian S, Malekhosseini SA. Alveolar echinococcosis of the liver: report of three cases from different geographic areas of iran. Hepat Mon. 2012;12(9):6143. doi: 10.5812/hepatmon.6143. [PubMed: 23087758].

10. Geramizadeh B, Attaran Y, Malek-Hosseini SA, Kaviani MJ, HossieniAsl K. Photoclinic. Alveolar hydatid cyst of the liver. Arch Iran Med. 2011;14(3):211-2. doi: 011143/AIM.0016. [PubMed: 21529115].

11. Masoon SHF, Sharifi N. Alveolar hydatid disease. A case report. Med J Islamic Rep Iran. 2007;20:205-7.
12. Farrokh D, Zandi B, Pezeshki Rad M, Tavakoli M. Hepatic alveolar echinococcosis. Arch Iran Med. 2015;18(3):199-202. doi: 0151803/AIM.0013. [PubMed: 25773697].

13. Maddah G, Abdollahi A, Sharifi-Nooghabi R, Tavassoli A, RajabiMashadi MT, Jabbari-Nooghabi A, et al. Difficulties in the diagnosis and management of alveolar hydatid disease: A case series. Caspian JIntern Med. 2016;7(1):52-6. [PubMed: 26958334].

14. Raiesolsadat MA, Mirsadeghi SA, Yaghoubi MA, Elahifar A, ZarifZakerian B. Alveolar hydatid cyst: A case report. ZJRMS. 2010;12:49-52.

15. Brunetti E, Kern P, Vuitton DA, Writing Panel for the Who-Iwge Expert consensus for the diagnosis and treatment of cystic and alveolar echinococcosis in humans. Acta Trop. 2010;114(1):1-16. doi 10.1016/j.actatropica.2009.11.001. [PubMed:19931502].

16. Tappe D, Frosch M. Rudolf Virchow and the Recognition of Alveolar Echinococcosis. 1850s Emerg Inf Dis. 2007;13:732-5.

17. Knapp J, Sako Y, Grenouillet F, Bresson-Hadni S, Richou C, GbaguidiHaore $\mathrm{H}$, et al. Comparison of the serological tests ICT and ELISA for the diagnosis of alveolar echinococcosis in France. Parasite. 2014;21:34. doi: 10.1051/parasite/2014037. [PubMed: 25058754].

18. Cai DM, Wang HY, Wang XL, Jiang Y, Luo Y, Li YZ. Ultrasonographic findings of small lesion of hepatic alveolar echinococcosis. Acta Trop. 2016 doi: 10.1016/j.actatropica.2016.01.030. [PubMed: 26892870].

19. Liu W, Delabrousse E, Blagosklonov O, Wang J, Zeng H, Jiang Y, et al. Innovation in hepatic alveolar echinococcosis imaging: best use of old tools, and necessary evaluation of new ones. Parasite. 2014;21:74. doi: 10.1051/parasite/2014072. [PubMed: 25531446].

20. Goel R, Kuganolipava S, Krokiadis M, Wilkinson M. Cyclist: slightly foxed. BMJ Case Rep. 2013;2013 doi:10.1136/bcr-2013-008964. [PubMed: 23580683].

21. McManus DP, Li Z, Yang S, Gray DJ, Yang YR. Case studies emphasising the difficulties in the diagnosis and management of alveolar echinococcosis in rural China. Parasit Vectors. 2011;4:196. doi 10.1186/1756-3305-4-196. [PubMed: 21981994].

22. Li F, Yang M, Li B, Yan L, Zen Y, Wen T, et al. Initial clinical results of orthotopic liver transplantation for hepatic alveolar echinococcosis. Liver Transpl. 2007;13(6):924-6. doi: 10.1002/lt.21187. [PubMed: 17538987].

23. Wang $\mathrm{H}$, Lu C, Liu X, Zhang W. Metastatic and prognostic factors in patients with alveolar echinococcosis. Int J Clin Exp Pathol. 2015;8(9):11192-8. [PubMed: 26617841]. 